\title{
Myositis ossificans of the masticatory muscle monitored over three generations: A case report and review of the literature
}

\author{
CHENLIN LI, CHI YANG, WEILIU QIU, YANGTING QIU, QIAN JIANG and MINJIE CHEN \\ Department of Oral Surgery, Ninth People's Hospital, Shanghai Jiao Tong University School of Medicine, \\ Shanghai Key Laboratory of Stomatology and Shanghai Research Institute of Stomatology, \\ National Clinical Research Center of Stomatology, Shanghai 200011, P.R. China
}

Received June 19, 2019; Accepted November 15, 2019

DOI: $10.3892 /$ etm.2020.8491

\begin{abstract}
Myositis ossificans (MO) is a rare disease and its major feature is the formation of heterotropic bone involving muscle or any other type of soft tissue (tendons, ligament, fascia and connective tissue). In the present study, a case report of a patient diagnosed with MO is presented. The diagnosis was established by evaluation of the medical history of the patient and the patient's family, as well as clinical data, radiology and post-operative pathology. The patient underwent excision surgery of the calcified lesion. In addition, genomic DNA was examined from blood samples of the patient and the patient's father with their consent. A mutation in the non-coding region was detected but any direct causative effect remains elusive. The present case report provided significant information with regard to the incidence of $\mathrm{MO}$ in four members of the same family assessed over three generations. The disease exhibited a unique localization in the maxillofacial region.
\end{abstract}

\section{Introduction}

Myositis ossificans (MO) is a disease featuring heterotropic bone formation within a muscle or any other type of soft tissue (tendons, ligament, fascia and connective tissue). MO is divided into two categories as follows: MO progressiva (MOP) or fibrodysplasia ossificans progressive (FOP) and MO traumatica (MOT) $(1,2)$.

MO encountered in the masticatory muscles is not frequently reported and its most common symptom is

Correspondence to: Dr Qian Jiang or Dr Minjie Chen, Department of Oral Surgery, Ninth People's Hospital, Shanghai Jiao Tong University School of Medicine, Shanghai Key Laboratory of Stomatology and Shanghai Research Institute of Stomatology, National Clinical Research Center of Stomatology, 639 Zhi-Zao-Ju Road, Shanghai 200011, P.R. China

E-mail: jiangqian0720@hotmail.com

E-mail: chenminjie00@126.com

Key words: myositis ossificans, masticatory muscles, pedigree, mutation trismus $(3,4)$. A case of MO in the masticatory muscles was described in the present study that was performed over three generations.

\section{Case report}

A 22-year-old male was admitted to the Department of Oral Surgery, Ninth People's Hospital, Shanghai Jiao Tong University School of Medicine (Shanghai, China) in March 2013 with an inability to open his mouth which had been developing gradually for $>3$ years. The patient was previously diagnosed with pericoronitis of the lower left wisdom tooth. Following treatment for this condition, the patient noted a progressive decrease in the ability to open his mouth, resulting in almost complete trismus. In December 2011, coronoidectomy on the left side and extraction of the left lower and upper molars were performed. The patient's active maximal incisal opening (MIO) gradually decreased post-operatively and trismus reappeared following 1 month from the date of the surgery (Fig. 1).

Physical examination revealed a well-nourished male without apparent developmental abnormalities. The MIO at presentation was $0 \mathrm{~mm}$. The patient was unable to protrude his mouth or produce any excursive movements. Palpation of the masseter and temporalis muscles did not reveal any abnormality. Intraoral examination indicated normal mucosa with no evidence of submucous fibrosis.

CT scans indicated a region of high attenuation within the left lateral pterygoid muscle, extending from the left lateral pterygoid plate to the medial surface of the left mandible (Fig. 2). Laboratory test results, including calcium, phosphate and parathyroid hormone, were within normal limits.

Following review of the patient's family history, a pedigree was established in which the possible occurrence of the disease was depicted over three generations (Fig. 3). Apart from the proband, three other members of the family had been similarly affected. The involvement was exclusively localized to the maxillofacial region, while hands and feet were normal. However, imaging characteristics, treatment options and prognosis were different among the patients. The patient's grandmother and aunt noted limited mouth opening without apparent trauma or infection and the sign spontaneously resolved over 1 year. The patient's sister has never suffered 


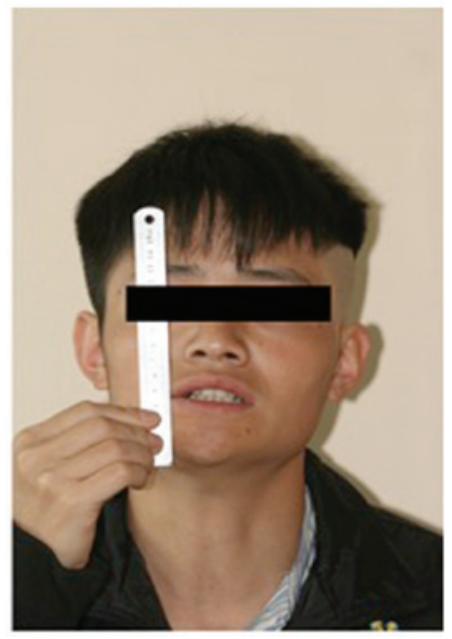

Figure 1.Pre-operative image of the patient exhibiting limited mouth opening. The maximum mouth opening capacity of the patient is demonstrated in the image.

from limited mouth opening. In comparison, the situation was different in the patient's father: First, the wisdom tooth extraction was performed due to symptoms associated with MIO occurring, which then further worsened. Furthermore, the lesion did not regress and it became a functional handicap-trismus. In addition, no calcifications were observed in the masticatory muscles as determined by CT scans (Fig. 4) and histology. The MIO of the proband's father was $2.5 \mathrm{~cm}$ following coronoidectomy and masticatory muscle stripping.

The ossification of the lateral pterygoid muscle of the proband examined in the present study led to the diagnosis of MO. However, the classification of the patient's MO type (MOP or MOT) remained undetermined. Mutational analysis is usually applied to confirm the diagnosis of MOP. It may aid the early diagnosis of this disease and is valuable for cases of suspected variants. The proband, the proband's father and the proband's sister were willing to participate and genomic DNA was examined from their blood. Mutations in the activin A receptor type 1 (ACVR1) gene may be screened by PCR amplification of 12 exons containing protein-coding sequences. The results indicated a mutation but it was after the stop-codon in the non-coding region (Fig. 5). However, the disease was finally diagnosed as MOP due to the patients' medical and family history, as well as clinical, radiological and pathological results.

During surgery of the proband in 2013, the calcified lesion was excised and the masticatory muscle was removed. The excised site was filled with a buccal fat pad. Post-operative pathology indicated that bone tissue was formed and that the trabeculae of the bone tissues contained osteocytes and numerous reversal lines (Fig. 6). CT examination of the pterygoid lamina to the internal ramus indicated no calcification and band formation at 2 months after the surgery. At 2 years after surgery, the patient's MIO is maintained at $1.5 \mathrm{~cm}$ (Table I).

\section{Discussion}

MO of masticatory muscles is not frequently reported in the literature with the most common symptom of being trismus. Boffano et al (5) reviewed 42 cases of MO of the

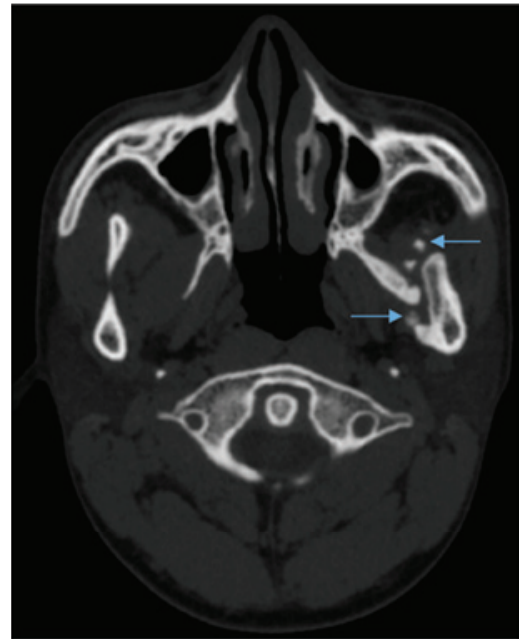

Figure 2. Pre-operative horizontal plane CT scan. Arrows indicate heterotopic bone formation in the left lateral pterygoid muscles.

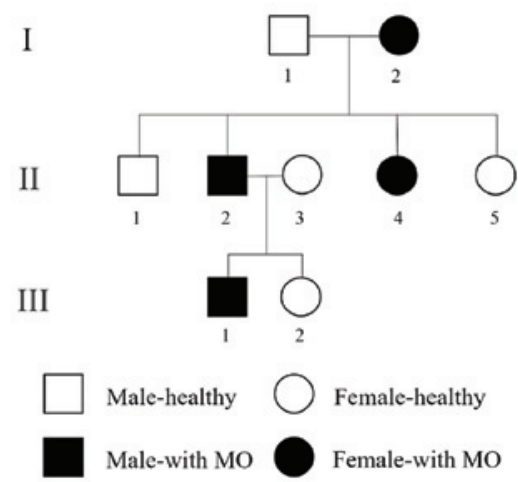

Figure 3. Pedigree of the proband (the third generation, no. 1) with MO over three generations, indicating that four family members are affected. MO, myositis ossificans.

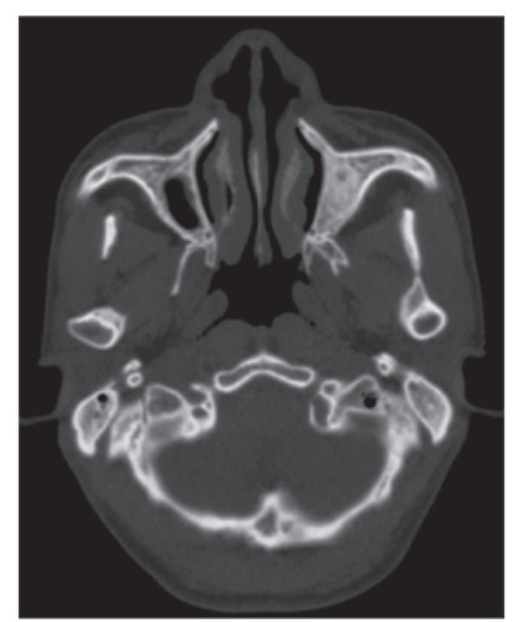

Figure 4. Absence of calcifications in the proband's fathers' masticatory muscles (pre-operative CT scan, horizontal plane).

masticatory muscles, among which the masseter was the most frequently involved muscle (25 patients, $47 \%$ ), followed by the medial pterygoid muscle (14 patients, 26\%), the lateral 


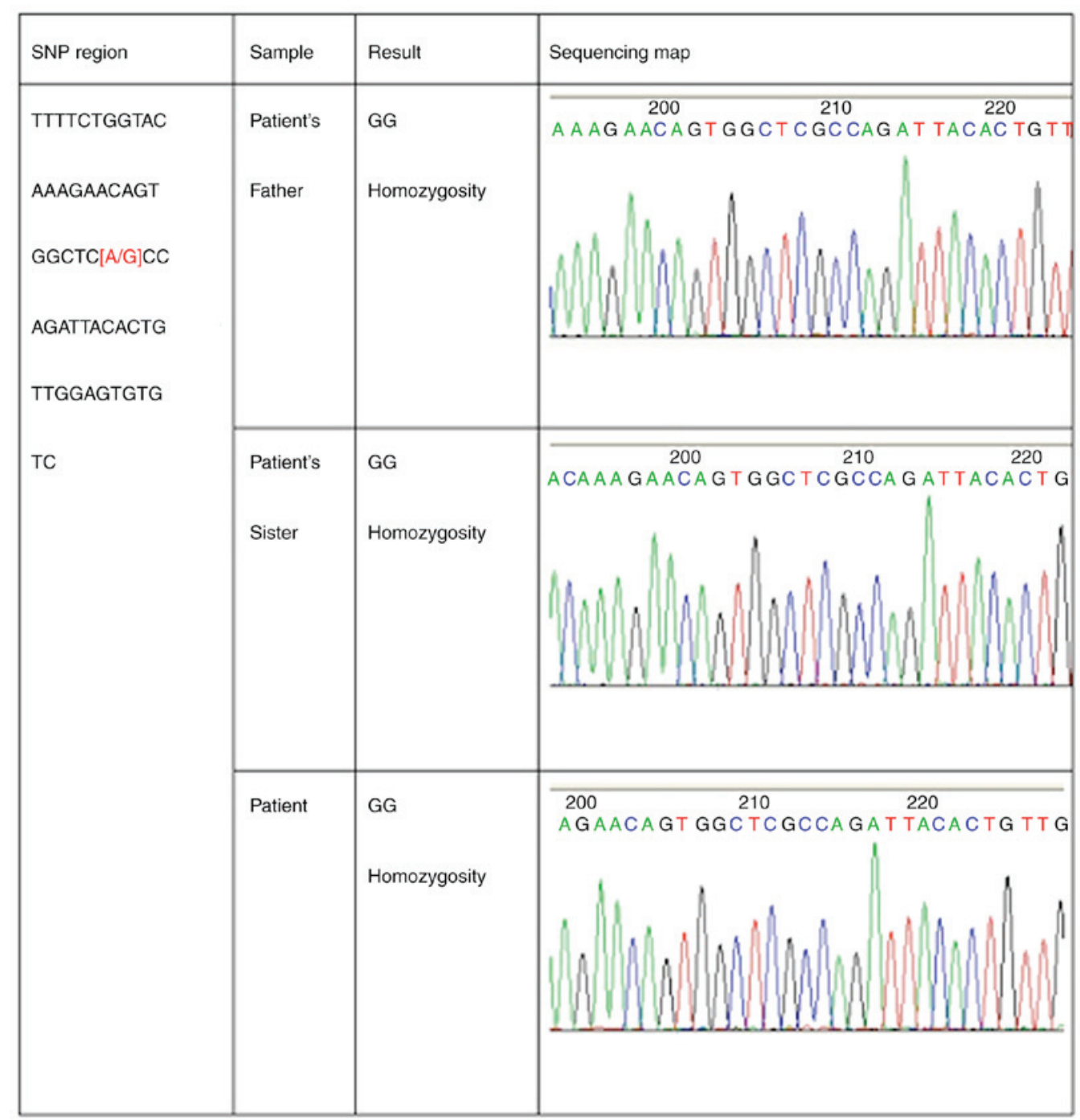

Figure 5. Absence of mutations in the samples. SNP, single nucleotide polymorphism.

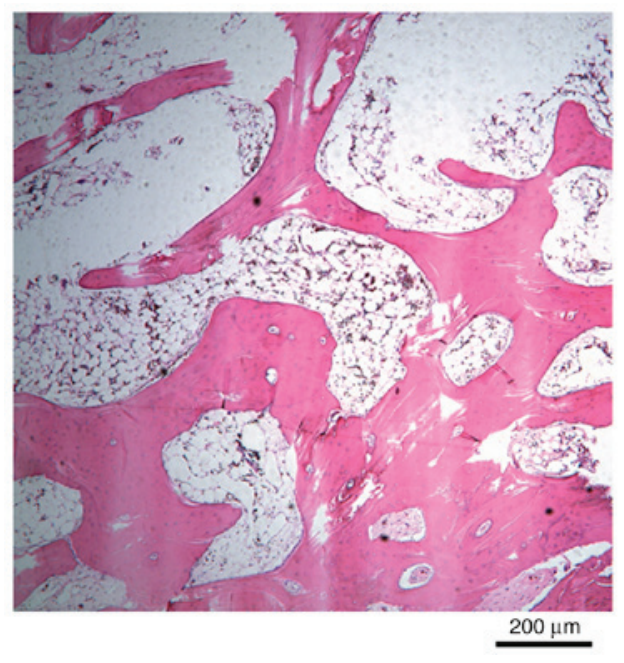

Figure 6. Histology demonstrates trabeculae of bone tissue with osteocytes and numerous reversal lines (HE staining; magnification, x200; scale bar, $200 \mu \mathrm{m})$. HE, hematoxylin-eosin staining.

pterygoid muscle (9 patients, 17\%) and the temporalis muscle (5 patients, 10\%).
CT is considered the best way to characterize the calcification zone pattern and may be used prior to determination of the characteristic calcification mode on radiography (6). Early lesions of the axial skeleton have been reported to appear as amorphous calcifications within the soft tissue. The accumulation of mature lesions may appear well-circumscribed with a ring of calcification surrounding a relatively radiolucent central portion (7). Therefore, long-standing lesions may appear with diffuse calcification (7).

The histological course of MO progresses from an immature, highly cellular fibroblastic lesion to a mature mass with a peripheral lamellar bone. The hallmark of MO is the manifestation of the zonal architecture with peripheral ossification and the presence of a central cellular region. The outer zone is composed of a mature lamellar bone with active osteoclasts, whereas the intermediate zone occasionally includes osteoid tissue, cartilage or woven bone formation and active osteoblasts. The central zone is composed of loose fibrovascular tissue resembling granulation tissue, containing spindle cells and prominent giant mesenchymal cells $(1,8)$.

In 1998, Debeney-Bruyerre et al (9) reported similar cases to those of the present study. The proband's mouth opening was limited and devoid of congenital malformations. A CT 
scan demonstrated the formation of bilateral masticatory muscle calcification and similar symptoms were noted in the proband's pedigree for five consecutive generations. The diagnosis of this disease was FOP. Unfortunately, no subsequent genetic linkage studies were performed, since the patient and his relatives did not cooperate.

In 2006, Shore et al (10) successfully mapped FOP to chromosome 2q23-24. Typical patients with FOP (congenital double-knee malformation and progressive heterotopic ossification) developed this disease due to mutations in the ACVR1 gene, which affected the bone morphogenetic protein (BMP) type I receptor. When the 617th guanine is mutated into adenine $(617 \mathrm{G} \rightarrow \mathrm{A})$, arginine becomes histidine during protein translation, exposing the original phosphorylation site of this protein. BMP binds to the mutated residue, which in turn activates the intracellular signaling pathway and promotes the formation of heterotopic bone.

Subsequently, Kaplan et al (11) performed a DNA sequence analysis of 112 patients with FOP and demonstrated that in addition to the classical frequent mutation sites reported previously, new mutation sites of ACVR1 were present in a limited number of atypical patients with FOP.

A combined analysis of the clinical characteristics of this proband with previous data from cases reported in the literature was performed in order to confirm the diagnosis. Mutational analysis of the ACVR1 gene was performed. Pathogenic gene analysis was performed using the samples from the patient's father and the patient's sister. Written informed consent was provided by all participants of the study.

ACVR1 is divided into four parts, namely the ligand region, the transmembrane region, the glycine-serine enrichment region and the kinase region. Initially, blood samples from the patient, the patient's father and the patient's sister were analyzed for frequent mutation sites of the ACVR1 gene. The test results indicated no mutation in the 617th guanine of these three subjects. Subsequently, the entire exon of the ACVR1 gene was expanded and a hybrid region including thymine and cytosine was identified in exon 12; this mutation was located in the non-coding region following the stop codon. Whether the mutation has an impact on the disease reported in the current study remains elusive and may be worthy of investigation in the future. Therefore, mutations in the ACVR1 gene were not detected in the pedigree analyzed in the present study. Whole-genome sequencing, including coding and non-coding regions, will be performed in future studies.

The treatment of MO is controversial and challenging. The patient of the present case report underwent two surgeries as follows: Coronoidectomy on the left side in 2011 and excision of calcified lesion and buccal fat pad reconstruction in 2013. Complete excision of the ossified mass appears to be a generally accepted treatment for this disease (12). Following removal of the ossified mass, inter-positional fat graft is used as a means of reconstruction by a limited number of surgeons (13). Active physiotherapy may be initiated in the immediate post-operative period to sustain the mouth opening and prevent scar formation (14). Non-surgical treatments include non-steroidal anti-inflammatory drugs, magnesium, bisphosphonates and warfarin. Furthermore, low-dose radiation therapy, immobilization, ice, elevation, ultrasound, cold laser and iontophoresis may be applied (15). 


\section{Acknowledgements}

Not applicable.

\section{Funding}

No funding was received.

\section{Data availability}

The datasets used and/or analyzed during the present study are available from the corresponding author on reasonable request.

\section{Authors' contributions}

QJ and CL wrote the manuscript. WQ and CY were responsible for patient diagnosis and treatment. CY, MC and YQ performed the surgery. MC revised the manuscript. CL has made substantial contributions to analysis and interpretation of medical records, including genomic DNA examination; and has drafted the manuscript for important intellectual content, including case report, discussion and literature review. QJ has made substantial contributions to conception and design, and acquisition of medical records.

\section{Ethics approval and consent to participate}

Written informed consent was provided by all participants of the study.

\section{Patient consent for publication}

The patients provided their consent for the publication of the data and images in the present study.

\section{Competing interests}

The authors declare that they have no competing interests.

\section{References}

1. Walczak BE, Johnson CN and Howe BM: Myositis ossificans. J Am Acad Orthop Surg 23: 612-622, 2015.
2. Smith R: Myositis ossificans progressiva: A review of current problems. Semin Arthritis Rheum 4: 369-380, 1975.

3. Godhi SS, Singh A, Kukreja P and Singh V: Myositis ossificans circumscripta involving bilateral masticatory muscles. J Craniofac Surg 22: e11-e13, 2011.

4. Parkash $\mathrm{H}$ and Goyal M: Myositis ossificans of medial pterygoid muscle. A cause for temporomandibular joint ankylosis. Oral Surg Oral Med Oral Pathol 73: 27-28, 1992.

5. Boffano P, Zavattero E, Bosco G and Berrone S: Myositis ossificans of the left medial pterygoid muscle: Case report and review of the literature of myositis ossificans of masticatory muscles. Craniomaxillofac Trauma Reconstr 7: 43-50, 2014.

6. Shehab D, Elgazzar AH and Collier BD: Heterotopic ossification. J Nucl Med 43: 346-353, 2002.

7. Kim DD, Lazow SK, Har-El G and Berger JR: Myositis ossificans traumatica of masticatory musculature: A case report and literature review. J Oral Maxillofac Surg 60: 1072-1076, 2002.

8. Saka B, Stropahl G and Gundlach KK: Traumatic myositis ossificans (ossifying pseudotumor) of temporal muscle. Int J Oral Maxillofac Surg 31: 110-111, 2002.

9. Debeney-Bruyerre C, Chikhani L, Lockhart R, FavreDauvergne E, Weschler B, Bertrand JC and Guilbert F: Myositis ossificans progressiva: Five generations where the disease was exclusively limited to the maxillofacial region. A case report. Int J Oral Maxillofac Surg 27: 299-302, 1998.

10. Shore EM, Xu M, Feldman GJ, Fenstermacher DA, Cho TJ, Choi IH, Connor JM, Delai P, Glaser DL, LeMerrer M, et al: A recurrent mutation in the BMP type I receptor ACVR1 causes inherited and sporadic fibrodysplasia ossificans progressiva. Nat Genet 38: 525-527, 2006.

11. Kaplan FS, Xu M, Seemann P, Connor JM, Glaser DL, Carroll L, Delai P, Fastnacht-Urban E, Forman SJ, GillessenKaesbach G, et al: Classic and atypical fibrodysplasia ossificans progressiva (FOP) phenotypes are caused by mutations in the bone morphogenetic protein (BMP) type I receptor ACVR1. Hum Mutat 30: 379-390, 2009.

12. Hanisch M, Hanisch L, Fröhlich LF, Werkmeister R, Bohner L and Kleinheinz J: Myositis ossificans traumatica of the masticatory muscles: Etiology, diagnosis and treatment. Head Face Med 14: 23, 2018.

13. Rattan V, Rai S and Vaiphei K: Use of buccal pad of fat to prevent heterotopic bone formation after excision of myositis ossificans of medial pterygoid muscle. J Oral Maxillofac Surg 66: 1518-1522, 2008.

14. Kayal L, Manoharan GVMG and Joshi B: Myositis ossificans of the masseter muscle. Saudi J Med Med Sci 6: 119-120, 2018.

15. Thangavelu A, Vaidhyanathan A and Narendar R: Myositis ossificans traumatica of the medial pterygoid. Int J Oral Maxillofac Surg 40: 545-549, 2011.

This work is licensed under a Creative Commons Attribution-NonCommercial-NoDerivatives 4.0 International (CC BY-NC-ND 4.0) License. 